Review Article

\title{
Diffusion-weighted MRI: role in the differential diagnosis of the brain tumors
}

\begin{abstract}
Diffusion-weighted MRI (or diffusion-weighted imaging, DWI) is a method that is based upon random (Brownian) movements of water molecules. In diffusion-weighted imaging, additional gradients (which are the strong magnetic fields) are applied in very short durations. If restricted diffusion is present, increased signal is obtained. However, the diffusion-weighted images also have $\mathrm{T} 2$ characteristics and therefore, lesions that are very bright on $\mathrm{T} 2$ weighted images may still appear bright on diffusion weighted sequences. To overcome this "T2 shine-through" effect, apparent diffusion coefficient (ADC) maps are produced by getting three orthogonal measurements, taking the average, for each voxel. ${ }^{1}$ Areas with restricted diffusion appears dark on ADC images.

DWI is mostly used in the evaluation of strokes, since the ischemia causes restricted diffusion within the cells. This is mainly due to the disruption of $\mathrm{Na} / \mathrm{K}$ ATP as epump within the cellular membrane, which then causes the accumulation of sodium, and therefore water, within the cell. This edema is called "cytotoxic" edema. DWI is theme thod that shows acute infarction faster than the other MRI sequences. It also allows differentiation of cytotoxic and vasogenic edemas within brain tissue. Since the method is fast and does not need exogenous contrast material, it is widely used routine lyin MRI practice. Its applications are not limited to brain, it is also used in head \& neck and body imaging. In addition to infarctions, tumors, especially cellular tumors with high nucleus/cytoplasmic ratios may also show restricted diffusion. Therefore, DWI plays an important role in the differential diagnosis of brain masses. Examples of brain tumors that may show restricted diffusion are as follows:
\end{abstract}

Keywords: epidermoid cysts, meningiomas, chordomas, lymphomas, glial tumors, abscess
Volume 2 Issue 2 - 2015

Kamil Karaali, Akin Bayrak

Department of Radiology, Turkey

Correspondence: Kamil Karaali, Department of Radiology, Akdeniz University Hospital, Antalya, Turkey, Emailkamilkaraali@gmail.com

Received: February 28, 2015 | Published: March 19, 2015

\section{Epidermoid cysts}

These lesions are benign and non-neoplastic congenital or acquired cysts. Since they contain dense fluid with epidermal elements, they show restricted diffusion. DWI allows them to be differentiated from other cysts, especially the arachnoid cyst, which do not show any restricted diffusion (Figure 1). Their appearance on DWI are very characteristic so diagnosis is almost certain. ${ }^{2}$ DWI is also useful for detection residual tumors after operation.

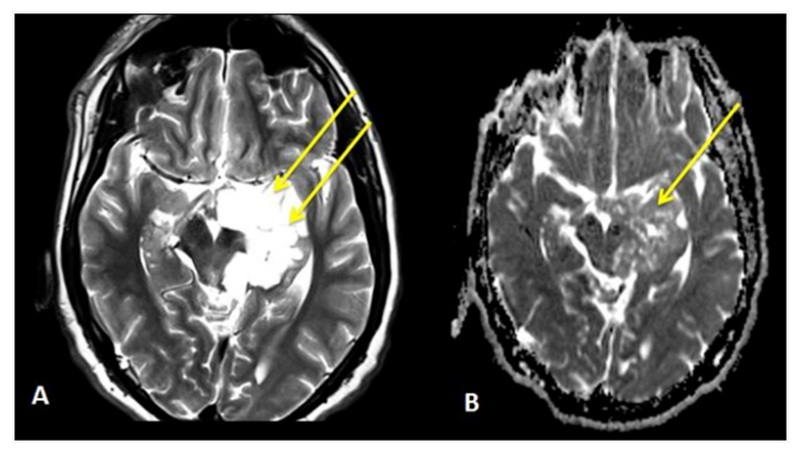

Figure I Epidermoid cyst. Lesion is very bright on T2-weighted image ( $A$, arrows), on ADC map (B) it is as dark as brain parenchyma due to the restricted diffusion.

\section{Meningiomas}

These are the most frequently observed extra axial masses. Their typical appear acnes make it easier to be diagnosed on routine MR images. They show intense enhancement after contrast, have extra axial mass characteristics and "dural tail" which is highly suggestive for meningiomas. Their subtypes are typical, atypical and malignant meningiomas. A typical and malignant meningiomas show much more prominent restricted diffusion (Figure 2). ${ }^{3}$

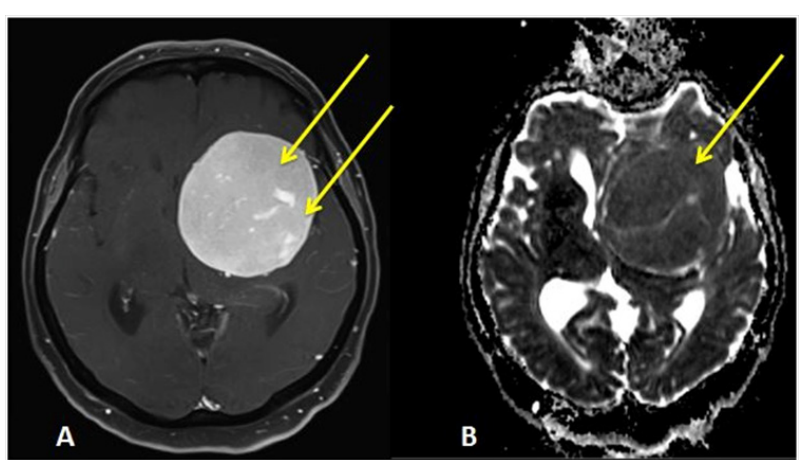

Figure 2 Meningioma. Post-contrast TI-weighted image (A) shows intense enhancement of the tumor (arrow). On ADC map, the mass is dark, due to the restricted diffusion. Pathology:Atipic meningioma,WHO grade 2.

\section{Chordomas}

Chordomas and chondro-sarcomas are rare primary bone tumors which involves skull base, especially the clivus. Imaging appear ances are usually similar. DWI may help differentiating chordomas, ${ }^{4}$ since especially the poorly differentiated chordomas show much more restricted diffusion than chondro-sarcomas (Figure 3). Chordomas may also be seen in sacrococcigeal region. 


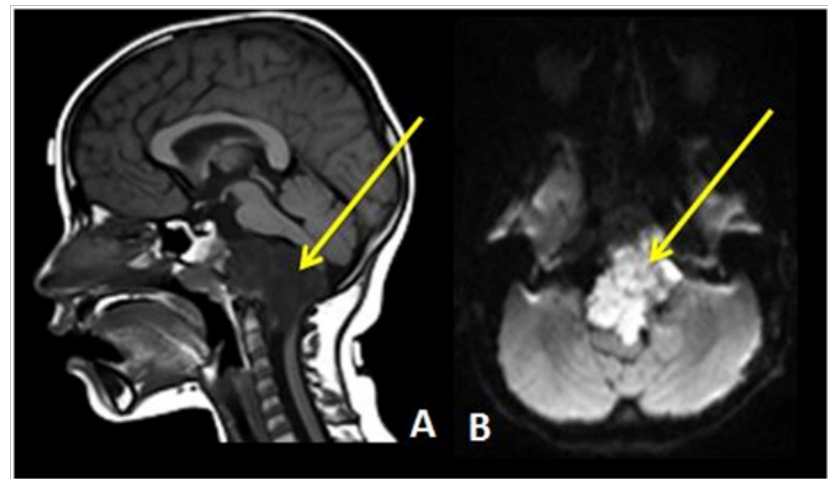

Figure 3 A 7-year-old male presented with difficulty in walking. Sagittal TI-weighted image shows destructive mass within clivus (arrow), which compresses brain stem. On DWI (B) brightness of the tumor shows restricted diffusion. Pathology: Chordoma.

\section{Lymphomas}

Primary central nervous system lymphomas are usually B-cell lymphomas. They usually involve periventricular/sub ependymal areas of the brain. They show intense enhancement after intravenous contrast material. Their diffusion is more restricted, ${ }^{5}$ compared to glial tumors (Figure 4). Also, they show lower perfusion than glial tumors.

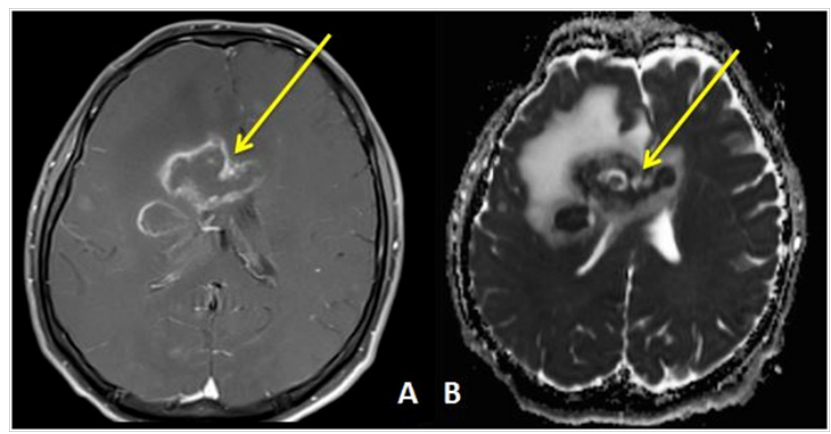

Figure 4 Lymphoma. A mass involving right frontal lobe and crossing the midline via corpus callosum. On post-contrast TI-weighted image (A) peripheral enhancement is seen (arrow). On ADC map (B) dark areas represent restricted diffusion.

\section{Glial tumors}

These are the most frequent primary brain tumors in adults. Grading is based on pathologic features. High grade tumors shown eovascularity and necrosis. On MRI, peripheral enhancement, irregularity and central necrosis are the characteristic findings. As the grade increases, degree of restricted diffusion within solid portion of the tumor also increases (Figure 5). On DWI, high grade tumors may also be differentiated from metastases, ${ }^{6}$ High grade tumors also show markedly increased perfusion, due to neovascularity.

\section{Abscess}

Although they are not neoplastic masses, abscess sometimes can be mistaken as necrotic tumors on imaging, since they both show peripheral contrast enhancement. Clinical presentation is important for the differential diagnosis. On MRI, liquid content of the abscesss how markedly restricted diffusion, ${ }^{7}$ which is extremely helpful for the diagnosis (Figure 6).

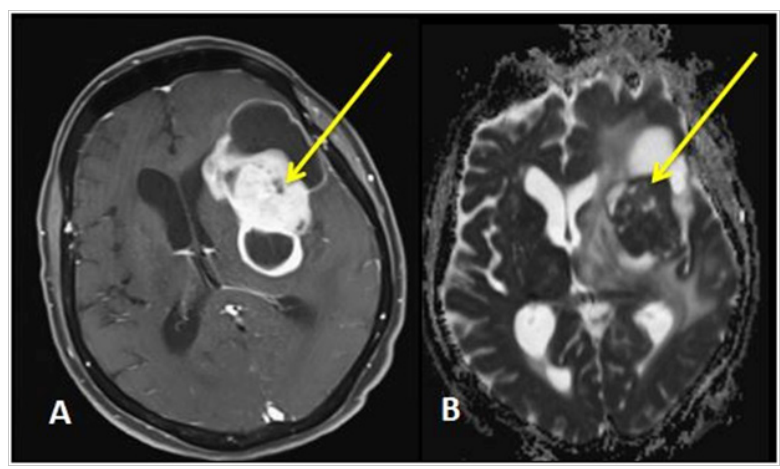

Figure 5 Glioblastome multiforme. Post-contrast TI-weighted image (A) shows intensely enhancing solid mass (arrow) with peripheral cystic components. On ADC map (B), solid portion shows restricted diffusion (arrow).

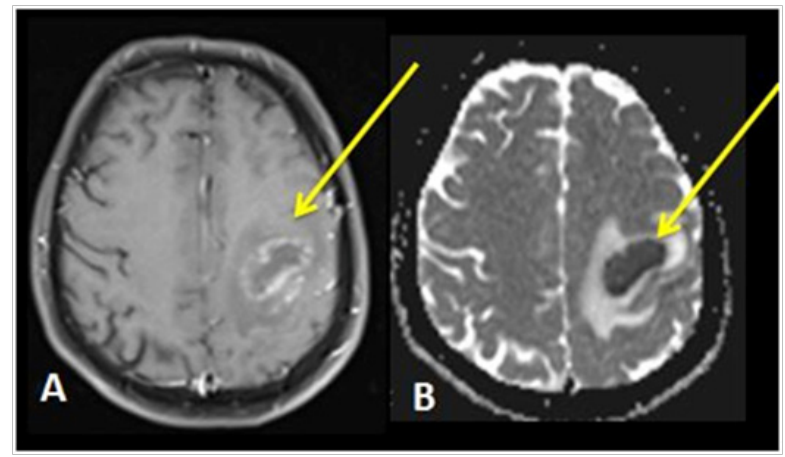

Figure 6 Abscess. Left parietal mass lesion showing peripheral contrast enhancement ( $A$, arrows). On ADC map (B), central portion of the mass hows restricted diffusion, which is typical for abscess.

\section{Conclusion}

In conclusion, DWI is an important part of MR imaging for the evaluation of brain masses. It should be noted that DWI cannot be used alone. Data obtained from routine T1, T2 and FLAIR sequences as well as post contrast images should be evaluated altogether. If possible perfusion imaging should also be added to evaluate the vascularity of the mass.

\section{Acknowledgments}

None.

\section{Conflicts of interest}

Authors declare that there is no conflict of interest.

\section{References}

1. Hagmann P, Jonasson L, Maeder P, et al. Understanding diffusion MR imaging techniques: from scalar diffusion-weighted imaging to diffusion tensor imaging and beyond. Radiographics. 2006;26(Suppl 1):S205S223.

2. Hakyemez B, Aksoy U, Yildiz H, et al. Intra cranial epidermoid cysts: diffusion-weighted, FLAIR and conventional MR findings. Eur $J$ Radiol. 2005;54(2):214-220.

3. Nagar VA, Ye JR, Ng WH, et al. Diffusion-Weighted MR Imaging: Diagnosing Atypical or Malignant Meningiomas and Detecting Tumor Dedifferentiation. Am J Neuroradiol. 2008;29(6):1147-1152. 
4. Yeom KW, Lober RM, Mobley BC, et al. Diffusion-Weighted MRI: Distinction of Skull Base Chordoma from Chondro sarcoma. Am J Neuroradiol. 2013;34(5):1056-1061.

5. Kitis O, Altay H, Calli C, et al. Minimum apparent diffusion coefficients in the evaluation of brain tumors. Eur J Radiol. 2005;55(3):393-400.
6. Server A, Kulle B, Maehlen J, et al. Quantitative apparent diffusion coefficients in the characterization of brain tumors and associated peritumoral edema. Acta Radiol. 2009;50(6):682-689.

7. Xu XX, Li B, Yang HF, et al. Can diffusion-weightedimaging be used to differentiate brain abscess from other ring-enhancing brain lesions? A meta-analysis. Clin Radiol. 2014;69(9):909-915. 